\title{
TERAPIA OCUPACIONAL: LA ACTIVIDAD COMO HERRAMIENTA DE TRABAJO EN LA UNIDAD DE PATOLOGÍA DUAL DE AGUDOS
}

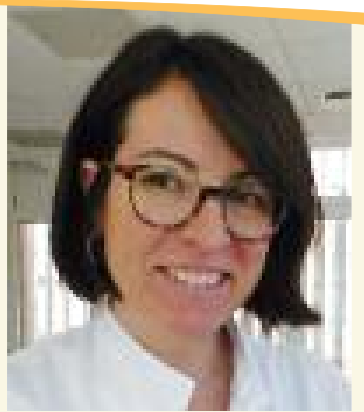

ZAIDA CAÑADAS ACEÑA

Terapeuta ocupacional. Unidad de Patología Dual de Agudos.

Institut de Neuropsiquiatria i

Addiccions.

Centre Dr. Emili Mira.

Parc de Salut Mar.

Santa Coloma de Gramanet (Barcelona).

\section{INTRODUCCIÓN}

Se denomina patología dual a la comorbilidad en un mismo individuo de un trastorno psiquiátrico y un trastorno por consumo de sustancias psicoactivas. Los expertos establecen que las personas con patología dual presentan mayor gravedad clínica y social, realizan más ingresos y presentan más riesgo suicida. También se observa una peor adherencia a los tratamientos respecto a los pacientes con presencia de un solo trastorno y una mayor utilización de los recursos sociales y sanitarios. Ante la elevada prevalencia y complejidad, así como la gravedad que presenta este colectivo, se recomienda que el abordaje terapéutico en patología dual tiene que tratar ambos trastornos de forma simultánea, desde el primer punto de contacto ${ }^{1}$ y con equipos multidis- ciplinares, además de con seguimientos y objetivos a largo plazo².

\section{TERAPIA OCUPACIONAL: APORTACIONES EN LA UNIDAD DE PATOLOGÍA DUAL DE AGUDOS}

Las unidades de hospitalización de patología dual de agudos (UPDA) dan asistencia a personas con patología dual en fase aguda, que, por las características clínicas o por la presencia de alteraciones graves del comportamiento, requieren de un tratamiento integrado en un régimen de ingreso y en un único dispositivo asistencial $^{3}$. La estancia media es de 15 a 21 días, y los primeros días de ingreso se caracterizan, en líneas generales, por la presencia de sintomatología propia del trastorno mental, así como de la asocia-

Correspondencia: Zaida Cañadas Aceña

Correo electrónico: zaida.canadas.to@hotmail.com 


\section{El abordaje terapéutico en patología dual tiene que tratar ambos trastornos de forma simultánea, desde el primer punto de contacto y con equipos multidisciplinares}

da a la retirada de sustancias (desintoxicación).

Integrada en la UPDA y formando parte del equipo multidisciplinar, encontramos la figura del terapeuta ocupacional. Las intervenciones que se realizan desde el programa de terapia ocupacional (TO) van dirigidas a la promoción del bienestar y la salud a través de la ocupación, y su principio básico es el mantenimiento o recuperación de autonomía personal.

A grandes rasgos, tanto el trastorno mental grave como la adicción a sustancias afecta a todos los niveles de desempeño ocupacional, alterando roles, rutinas, hábitos e intereses. El consumo de sustancias, además, marca rutinas dominantes, inhabilitando la capacidad de controlar la conducta y de realizar actividades alternativas al ciclo de consumo. La práctica diaria de nuestra profesión confirma las dificultades de este colectivo para adherirse a los tratamientos. De igual modo, observamos que las recaídas, tanto del trastorno mental como de la adicción, suelen producirse con cierta periodicidad y, por este motivo, es frecuente que los pacientes verbalicen sentimientos de desesperanza y creencias de incapacidad de cambio.
Según lo expuesto, ¿es posible que un paciente agudo y dual pueda beneficiarse de la intervención del terapeuta ocupacional en la UPDA? La respuesta es sí.

\section{METODOLOGÍA}

La propuesta de intervención desde el programa de TO se sustenta en bases teóricas que provienen de modelos propios de TO (Modelo de Ocupación Humana y Modelo Canadiense del Desempeño Ocupacional, entre otros), además de modelos que abordan el cambio de conductas adictivas (Modelo Transteórico del Cambio), o de métodos que nos aportan técnicas para favorecer el cambio (Entrevista Motivacional).

El plan de tratamiento es individualizado, significativo y motivador para el usuario con la finalidad de promover que sea la propia persona la que consiga producir el cambio en su desempeño ocupacional. No obstante, existen unos objetivos comunes a todos los pacientes ingresados y que estarán presentes en todas las propuestas de intervención: a) favorecer la conciencia de enfermedad, b) establecer el posicionamiento en relación con el consumo de sustancias y favorecer un cambio respecto a este, c) favorecer la adherencia al tratamiento y d) favorecer la vinculación a los dispositivos de derivación al alta.

Uno de los conceptos clave de la intervención recae en la voluntariedad de asistencia y participación del usuario en el programa de TO. Permitir la toma de decisiones y la autogestión del recurso (programa de TO) dentro de ambientes normativos y estructurados (unidad hospitalaria) promueve el desempeño de un rol activo del paciente y de corresponsabilidad durante el proceso de ingreso.

La alta rotación y el perfil de pacientes atendidos en las unidades de UPDA tiene como consecuencia la heterogeneidad de los grupos terapéuticos. En las sesiones grupales, conviven pacientes que no coinciden ni en momento de ingreso (crisis-estabilizaciónprealta), ni en objetivos personales, ni en régimen de ingreso, etc. Además, el consumo de sustancias añade más diversidad al grupo si cabe, ya que los posicionamientos y objetivos respecto al consumo variarán también en función de la persona. Esta circunstancia hará que el terapeuta ocupacional tenga que crear y diseñar actividades, de las cuales todos los asistentes puedan beneficiarse ${ }^{4}$.

La actividad con propósito - es decir, dirigida a objetivos predeterminados - es la herramienta fundamental de trabajo de los terapeutas ocupacionales. Evaluar, pre- 
venir, entrenar, reeducar o tratar son algunos de los objetivos que persiguen las actividades que configuran el programa de TO. Para la correcta elección de estas como parte del tratamiento, el terapeuta habrá realizado un análisis previo de todos los factores implicados en la actividad (demandas motoras, sensoriales, perceptuales, cognitivas, emocionales, sociales, de independencia, culturales, riesgos, etc.). El análisis de la actividad, la adaptación y la gradación de las actividades permite modificarlas de acuerdo con las necesidades del paciente y también del grupo.

Para que el usuario consiga producir el cambio en su desempeño ocupacional, el terapeuta ocupacional divide el tratamiento en diferentes fases ${ }^{5}$. A continuación, se muestra cada una de las fases y la relevancia que adquiere la actividad como herramienta de cambio ${ }^{6}$ en cada una de ellas:

- Fase de exploración: explorar y generar curiosidad son los objetivos principales de esta fase. Las actividades estarán relacionadas con intereses identificados durante la evaluación previa, serán conocidas por el usuario y nos aseguraremos de que pueda resolver con éxito los desafíos propuestos. Generar sentimientos de confianza y seguridad respecto a los espacios, las actividades, el grupo y el terapeuta son propios de este momento.

En esta etapa, es frecuente que se establezcan pactos entre paciente y terapeuta relacionados con la participación y los apoyos que se prestarán para el manejo del craving, la ansiedad, la sintomatología relacionada con el trastorno mental, o con dificultades personales que pueda identificar el paciente.

- Fase de competencia: en esta fase, las actividades siguen relacionadas con los intereses del paciente, pero se aumenta su grado de complejidad. El objetivo de esta fase se centra en que el paciente realice un entrenamiento de habilidades que le permitan conseguir un mejor desempeño ocupacional. La mejora de habilidades permite conseguir objetivos y éxitos, hecho que contribuye al aumento de la percepción de autoeficacia y autoestima. En esta fase, se reducen significativamente los apoyos prestados de forma progresiva y se refuerzan de for- ma positiva la mejora de habilidades y capacidad de desempeño.

- Fase de logro: en esta fase, el paciente es capaz de aceptar retos y desafíos, sin la necesidad de apoyos y con capacidad de gestionar de forma autónoma todos los requerimientos de la actividad.

Al finalizar las sesiones, se generan espacios para la reflexión, donde los pacientes analizan su desempeño durante la realización de las actividades. Estas reflexiones variarán totalmente en función del momento de ingreso. La descompensación psicopatológica de los primeros momentos impacta y condiciona «la capacidad de hacer», al igual que lo hace en días posteriores el proceso de estabilización. Por lo tanto, la actividad permite una aproximación al trastorno y a su evolución, a través de la experimentación y la vivencia y no tanto desde argumentos racionales como se haría, por ejemplo, en la psicoeducación. Asimismo, el terapeuta realiza una devolución individual y grupal, en la que se compartirá con los usuarios el desarrollo de la actividad, los objetivos específicos trabajados y la evolución observada4.

\section{CONCLUSIONES}

El aumento de pacientes «duales» es una realidad ya presente en el circuito de salud mental y en el de 